\title{
A Survey on Model-based Fault Detection Techniques for Linear Time-Invariant Systems with Numerical Analysis
}

\author{
Masood Ahmad ${ }^{1,2}$ and Rosmiwati Mohd-Mokhtar ${ }^{1 *}$ \\ ${ }^{1}$ School of Electrical and Electronic Engineering, Universiti Sains Malaysia, Engineering Campus, 14300 \\ Nibong Tebal, Pulau Pinang, Malaysia \\ ${ }^{2}$ Department of Electrical and Computer Engineering, COMSATS University Islamabad (Lahore Campus) \\ Lahore, Pakistan
}

\begin{abstract}
With the ongoing increase in complexity, less tolerance to performance degradation and safety requirements of practical systems has increased the necessity of fault detection (FD) as early as possible. During the last few decades, many research findings have been developed in fault diagnosis that addresses the issue of fault detection and isolation in linear and nonlinear systems. The paper's objective is to present a survey on various state-of-art model-based FD techniques developed for linear time-invariant (LTI) systems for the interested readers to learn about recent development in this field. Model-based FD techniques for LTI systems are classified as parameter-estimation methods, parityspace-based methods, and observer-based methods. The background and recent progress, in context to fault detection, of each of these methods and their practical applications are discussed in this paper. Furthermore, two different FD techniques are compared via analytical equations and simulation results obtained from the DC motor model. In the end, possible future research directions in model-based FD, particularly for the LTI system, are highlighted for prosperous researchers. A comparison and emerging research topic make this contribution different from the existing survey papers on FD.
\end{abstract}

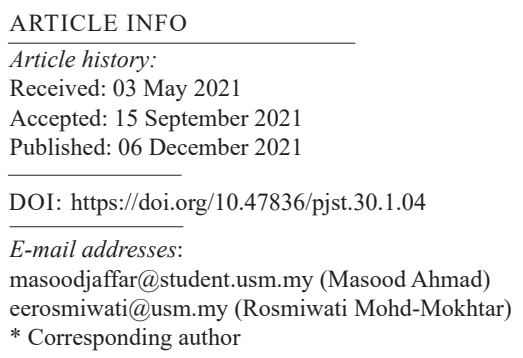

Keywords: Fault detection, Kalman filter, LTI system, model-based techniques, residual generation

\section{INTRODUCTION}

In the era of science and technology, every engineering system demands accuracy, reliability, and safety during its operation. However, to realize such systems, i.e., high- 
speed trains, power systems, aircraft, and chemical plants, increases the system complexity and financial cost. Moreover, any abnormal behavior in a safety-critical system causes performance degradation and leads to a dangerous situation. Thus, detecting and locating the fault early is important to ensure safety and reliability by taking necessary measures (Li et al., 2016; Franklin et al., 2019).

A fault is an unexpected event and input to the system that can occur in any part of the system. Concerning the location of occurrence, it is generally classified as an actuator fault (loss of control), a sensor fault (improper functioning of measuring components), and a component fault (variation in the system's internal parameters). Actuator and sensor faults can be considered additive faults, while component faults are multiplicative faults (Frank et al., 2000). Faults can also be classified according to their time behavior, i.e., abrupt fault, incipient fault, and intermittent fault. Any fault in a system causes poor performance and leads the entire system to collapse if it is not timely handled (Jie \& Patton, 2012; Liu et al., 2018; Na \& Ahmad, 2019).

The fault diagnosis system is composed of three sub-systems. Each subsystem is merged with the capabilities of detection, isolation, and identification (estimation) of the fault. FD is the first step in the fault diagnosis process that indicates the fault and its time of occurrence in the system. Fault isolation determines the location of a fault, and fault identification finds the type and size of a fault (Gao et al., 2015). Generally, fault diagnosis methods are model-based and data-driven methods depending on the system model information (Isermann, 1997). Data-driven-based FD methods solve the FD problem directly from online process data. Therefore, these techniques are more suitable for large-scale complex systems (Ding, 2014). On the other hand, model-based FD techniques utilize the analytical model of the process that reveals the physical meaning of process dynamics through the mathematical description. Ample of research has been done on model-based fault diagnosis and their applications on various linear and nonlinear systems during the last few decades (Gertler, 2017; Jie \& Patton, 2012; Isermann, 2006; Ding, 2013). Therefore, model-based techniques are chosen in many practical scenarios, provided that the analytical model of the process is well-established.

The core idea of the model-based FD technique is to reconstruct/estimate the output of a practical system using the analytical model, and the reconstructed output is compared with the actual output of the system measured from sensors. The difference between the two outputs is a residual signal, which indicates fault occurrence in the practical system. The model-based FD system is depicted in Figure 1. Residual evaluation refers to the process of extracting fault information from residuals to differentiate between fault and disturbance. Finally, a binary decision about the occurrence of a fault is made by comparing the evaluated residual signal with the pre-defined threshold. Hence, a model-based fault detection system consists of two subsystems: residual generation, residual evaluation, and 


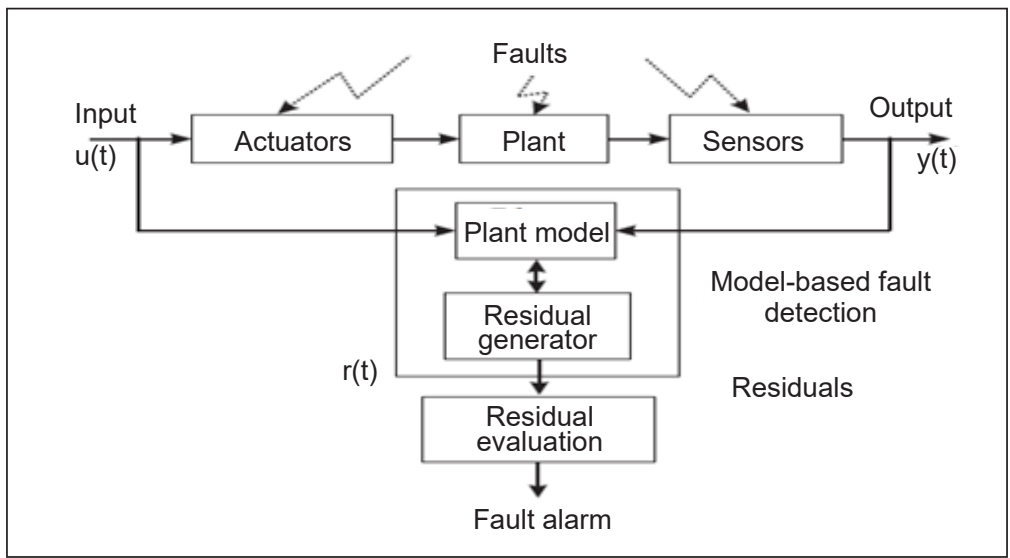

Figure 1. Model-based fault detection system (Chen et al., 2011)

threshold computation (Ding, 2013). In case of fault occurrence, an alarm is generated to intimate the operator, or some control action is taken to compensate the effect of fault for the smooth operation of an entire system. The process of modifying the control action according to fault nature is called fault-tolerant control (FTC). A detailed discussion on FTC is available in Zhang and Jiang (2008).

There are three main types of model-based fault detection techniques according to the way of residual generation. They are known as observer-based FD techniques, parityspace-based FD techniques, and parameter-estimation-based FD techniques (Isermann, 1984). Observer-based fault detection techniques correspond to the design of an observer for estimating system output and residual generation. In the parity-space-based approach, residual is generated by eliminating the initial states of dynamic systems and utilizing only system input and measurement data within a finite time window. The prime objective of both techniques is to ensure the robustness of residual against the process and measurement of unknown inputs. Finally, the parameter-estimation approach is used to detect the slight change/drift in the system parameters by comparing the actual parameters of the nominal process with the estimated parameters.

Considering the massive monetary losses caused by the faults, there is a need to find the solution for FD problems in safety-critical applications in the presence of unknown inputs and system parameter variations. External unknown inputs and parameter variations make the FD more intricated. In addition, FD becomes more complex in the closed-loop configuration because a fault in the system gets buried rapidly by the control actions. Most of the existing survey papers discussed the FD methods for nominal linear systems. This survey paper aims to look at another perspective, in which it presents state-of-art modelbased fault detection techniques recently developed for the linear time-invariant (LTI) systems in open and closed-loop configurations and subjected to unknown inputs and uncertainties simultaneously. Also, two well-known FD techniques, Kalman filter (Blanke 
et al., 2015) and optimal FD filter (Ding, 2013), are compared using an analogy in observer gain matrices and by simulations in this survey paper to demonstrate the clear picture of the FD process. Sensor and actuator fault in a linear model of DC motor is detected using both FD techniques. Their performance is compared in terms of detectability, computational burden, and design complexity.

Unlike other review papers that focused on the same subject, our paper also included a numerical comparison of fault detection techniques, and the simulation results were shown for verification. Furthermore, based on the review, future research direction in model-based fault detection approach is added to give the readers an idea and way forward to extend the research in this field. It is significant because the review outcome from this paper can be used as a reference for the readers of interest.

\section{LTI SYSTEM WITH DISTURBANCE AND FAULT}

LTI system subject to disturbance and fault can be expressed in the form of state-space representation as Equations 1 and 2:

$$
\begin{aligned}
& x(k+1)=A x(k)+B u(k)+E_{d} d(k)+E_{f} f(k) \\
& y(k)=C x(k)+D u(k)+F_{d} d(k)+F_{f} f(k)
\end{aligned}
$$

$x(k) \in R^{n}, u(k) \in R^{p}, y(k) \in R^{q}$ are state, input, output vectors respectively. $d \in R^{k} d_{\text {is }}$ $l_{2}$-norm bounded unknown input vector and $f \in R^{k_{f}}$ is $l_{2}$-norm bounded unknown fault vector. (A, B, C, D, $E_{d}, E_{f}, F_{d}, F_{f}$ ) are known matrices with appropriate dimensions. Furthermore, $E_{f}$ and $F_{f}$ represent the place where the fault occurs and its influence on the system dynamics (Ahmad et al., 2017). Component/process fault or modeling error in the system may cause the change of parameters of process dynamics. Equations 1 and 2 can be represented by incorporating the process fault (Equation 3).

$$
\begin{aligned}
& x(k+1)=(A+\Delta \mathrm{A}) x(k)+(B+\Delta B) u(k)+E_{d} d(k)+E_{f} f_{a}(k) \\
& y(k)=(C+\Delta C) x(k)+(D+\Delta D) u(k)+F_{d} d(k)+F_{f} f_{s}(k)
\end{aligned}
$$

Where $(\Delta A, \Delta B, \Delta C, \Delta D)$ represents the component faults/modeling errors. There are normally four types of sensor faults: sensor drift fault, sensor offset fault, fixed scaling factor fault, and sensor stuck fault. In addition, the same type of fault could be categorized for actuators (Franklin et al., 2019). It is important to mention here that this survey only focuses on additive fault detection techniques (sensor/actuator fault). 


\section{PARAMETER-ESTIMATION-BASED RESIDUAL GENERATION}

The core of the parameter-estimation technique is based on system identification by utilizing the system's measured input and output data. In this technique, system parameters of a practical system are identified either offline or online under the normal operating condition while assuming that the fault is reflected in the system's physical parameters. In the context of FD, residual is defined as a comparison between nominal parameters of the system in a fault-free case and estimated parameters. The estimated parameters should match with the system parameters in a fault-free situation. Any discrepancy in process parameters indicates the change/fault in the system. Parameters are estimated using parameterestimation algorithms, i.e., least squares (LS), recursive least squares (RLS), regularized LS, or extended least squares (ELS). These methods can be applied to any engineering system, provide the inherent information of system dynamics. The exploitation of these methods leads to an efficient fault detection and control system (Ding, 2013). Jesica and Poznyak (2018) developed a new technique using the Kalman filter and instrument variable method for parameter estimation in the stochastic system. The proposed technique minimized the influence of Gaussian noise and removed the biases in estimation, which remains available in standard least square methods. The designed scheme also improved the convergence speed.

Bachir et al. (2006) used the offline parameter-estimation technique for stator inter-turn short circuit fault and broken rotor bar detection. In this study, a new model of an induction machine for stator and rotor has been developed for fault detection. They introduced the new parameters in the original model of the induction machine for stator inter-turn fault detection and the design of a new faulty model for broken rotor bars detection.

Generally, the parameter-estimation technique requires one input and output signal, and it provides a more detailed picture of internal process quantities. Therefore, this technique is more suitable for component fault detection. However, it can be used for sensor/actuator fault detection as well. The major disadvantage is that it always needs an excitation signal for initiating the parameter-estimation process, which may not be suitable for the process, operating at a stationary point (Isermann, 2006). In addition, the parameter-estimation technique is less robust to unknown inputs that may affect the estimation process. Nevertheless, the performance of parameter-estimation-based- FD systems has been demonstrated by many successful applications in industrial processes and automatic control systems (Belmokhtar et al., 2015; Ye et al., 2015; Herrera \& Yao, 2018; Khang et al., 2018).

\section{PARITY-SPACE-BASED RESIDUAL GENERATION}

In a parity-space-based approach, a residual is generated by eliminating the effect of initial states of a dynamic system and utilizing only the system input and measurement data within 
a finite time window. The inconsistency arises in the residual in case of abnormal behavior evolving in the system dynamics. For example, the parity-space-based fault detection system has been studied (Sun et al., 2019; Zhang et al., 2006; Zhong et al., 2018). This section discusses parity-space-based FD for a linear discrete-time system in terms of design and implementation issues.

Consider Equation 1. The following parity relation can be established (Equation 4).

$$
y_{s}(k)=H_{o, s} x(k-s)+H_{u, s} u_{s}(k)+H_{d, s} d_{s}(k)+H_{f, s} f_{s}(k)
$$

Where $\theta_{s}(k)=\left[\theta^{T}(k-s), \theta^{T}(k-s+1), \ldots, \theta^{T}(k)\right]$, while $\theta=[y, u, d, f]$ and $s$ is the order of parity-space and

$$
H_{o, s}=\left[\begin{array}{c}
C \\
C A \\
\vdots \\
C A^{S}
\end{array}\right], H_{u, s}=\left[\begin{array}{cccc}
D & 0 & \ldots & 0 \\
C B & D & \ddots & \vdots \\
\vdots & \ddots & \ddots & 0 \\
C A^{S-1} B & \ldots & C B & D
\end{array}\right]
$$

$H_{d, s}, H_{f, s}$ can be obtained by substituting $\left(E_{d}, F_{d}\right)$ and $\left(E_{f}, F_{f}\right)$ in place of $(B, D)$ in $H_{u, s}$. Residual generator based on parity relation vector can be written as Equation 5.

$$
\begin{aligned}
& r(k)=M\left[y_{s}(k)-H_{u, s} u_{s}(k)\right] \\
& =M\left[H_{d, s} d_{s}(k)+H_{f, s} f_{s}(k)\right]
\end{aligned}
$$

$r(k)$ is a residual signal and $M$ is donated as a parity-space matrix, which contains a set of parity vectors and is defined as parity space, $P_{s k}=\left[M \mid M H_{o, s}=0\right]$. Let us denote $v_{s}$ is the parity vector and holds the condition $\left[v_{s} \in P_{s k}\right]$ then residual in Equation 5 can be represented as Equation 6.

$$
r(k)=v_{s}\left[H_{d, s} d_{s}(k)+H_{f, s} f_{s}(k)\right]
$$

Equation 6 clearly shows that parity relation-based residual design only requires the computation of parity vector $v_{s}$. In a fault-free case, $(f(k)=0), d_{s}(k)$ can be perfectly decoupled from the residual if the rank condition is satisfied $\left(M\left[H_{o, s} H_{d, s}\right]=0\right)$. However, the condition looks stringent and very hard to satisfy for practical systems. In such cases, the preferred solution for residual design is to apply the optimization technique to make an appropriate trade-off between robustness and sensitivity. For this purpose, several objective functions, with the prime aim to achieve the trade-off between robustness against disturbances and sensitivity to faults, have been defined. The following performance index is widely adopted for a parity-space-based residual generation (Equation 7) (Ding, 2013; Gertler, 2017). 


$$
J_{s}=\min _{s} \frac{\left\|v_{s} H_{d, s}\right\|_{2}}{\left\|v_{s} H_{f, s}\right\|_{2}}
$$

For successful fault detection, the $l_{2}$-norm of a residual signal in Equation 6 is the most commonly used evaluation function and is defined as Equation 8 (Ding, 2013).

$$
\|r(k)\|_{2}=\sqrt{\sum_{k=0}^{k=\infty} r^{T}(k) r(k)}
$$

A threshold can be set as $J_{t h}=\|d(k)\|_{2}^{2}=\delta_{d}\left(\delta_{d}\right.$ is an upper bound of disturbance energy) in a fault-free case. In the last step, the decision logic is used to declare the fault alarm.

An optimized parity-spaced-based fault detection algorithm was developed in (Odendaal \& Jones, 2014) for actuator fault detection in Meraka Modular UAV. The study optimized the parity relation, obtained from the standard parity-space approach, using the transformation matrix that forms the residual more sensitive to the fault. The said approach improved the computational burden compared to the online computation of covariance matrices at every instant in the Extended Kalman filter. As compared to FD techniques in an open-loop, fault detection in a closed-loop control system is much complicated because a closed-loop is more robust against exogenous inputs. External inputs surround a fault signal with low amplitude, and closed-loop control input makes the fault signal smaller. The phenomena reduce the fault detection performance and fault detection rate significantly. Sun et al. (2019) proposed a parity-space transformation-based fault detection system for the closed-loop control system. A stable kernel matrix for parity-space transformation was designed to obtain the more accurate parity-space in a closed-loop system that improves the fault detection performance. Furthermore, the fault detection rate has been improved by accumulating the residual in a time window.

Zhong et al. (2018) demonstrated an integrated design of residual generation and residual evaluation for fault detection in a linear discrete-time system subject to unknown input without complete knowledge of probability distribution. The study focuses on parityspace-based FD design to achieve an optimal trade-off between false alarm rate (FAR) and missed detection rate (MDR). Determination of parity-space vector and optimization of FAR and MDR are formulated in the minimum error minimax probability machine (MEMPM) framework. The proposed algorithm delivers an optimal trade-off between MDR and FAR in the worst-case scenario of unknown inputs without the information of stochastic distribution. 
Equation 6 demonstrates that the residual generator requires the past data of input and output measurements; hence, this residual generator is preferred for discrete-time dynamic systems only. Furthermore, it is important to mention that parity-space-based residual generators are more sensitive to unknown inputs because of their open-loop structure than the observer-based residual generator, which has a closed-loop configuration and is less sensitive to unknown input and system uncertainties. Despite the advantage of design simplicity, knowledge of previous data and constraints on parity-space order makes the parity-space-based residual generator non-ideal for online implementation. The solution to these two problems is a one-to-one mapping between the design parameters of the observer-based technique and the parity-space-based technique. This scheme is known as parity-space design, observer-based implementation (Ding, 2013; Isermann, 2006). By taking advantage of both designs, less design effort of parity-space vector, and online realization of the observer, this scheme can also be used for continuous-time systems.

The parity-space-based technique is well applied for FD purposes in the induction motor drive system (Dybkowski \& Klimkowski, 2017), electromechanical brake systems (Hwang \& Huh, 2015), vehicles (Wang et al., 2019), and power systems (Rasoolzadeh \& Salmasi, 2020).

\section{OBSERVER-BASED RESIDUAL GENERATION}

A survey on observer-based FD techniques for the LTI system is presented in this section. FD system based on Kalman filter is presented first for stochastic LTI systems with Gaussian noise. Next, unknown input observer (UIO) is discussed for perfectly decoupling the unknown input, followed by a discussion on optimal observer design for deterministic LTI systems subjected to norm-bounded unknown inputs and uncertainties.

\section{Kalman Filter-based Fault Detection}

A stochastic system is a dynamic system subjected to a stochastic/random noise, i.e., Gaussian noise with mean value and variance. The first stochastic fault detection system was developed using the innovations (residuals) generated by the Kalman filter (Mehra \& Peschon, 1971). A well-known Kalman filter, which looks like an observer in a deterministic environment, is used to estimate the system's state based on a series of measurements taken in time, having system inaccuracies and statistical noise. The residual signal contains the information of fault and the mutual effect of uncertainties, process, and measurement noise. Stochastic residual evaluation techniques are used to eliminate these undesired effects and recover the fault information from the residual. These techniques use the residual's statistical properties, i.e., mean, variance, and covariance, and try to detect statistical parameters change. Several statistical methods are available in the literature to evaluate 
the residual generated by the Kalman filter for fault detection purposes. A few of these methods are the chi-square test (Da \& Lin, 1996), multiple hypothesis tests (Bøgh, 1995), generalized likelihood ratio (GLR) test (Willsky \& Jones, 1976), and cumulative sum algorithms (Nikiforov et al., 1993).

Kalman filter provides an optimal estimate of system states, i.e., minimum covariance of error between estimated and actual states of the system. The unified approach is presented in Doraiswami and Cheded (2013) to detect and isolate the fault in a linear discrete-time system with measurement and system noise using the Kalman filter. Switched Kalman filter is designed for sensor fault detection and isolation in power converters (Kleilat et al., 2018). The proposed filter is an extended version of the standard Kalman filter combined with a disturbance decoupling observer and has confirmed satisfactory results disturbance. The incipient sensor fault detection in the continuous stirred-tank reactor benchmark process has been addressed in Gautam et al. (2017) using Kalman filter and GLR test. In this technique, signal to noise ratio (SNR) index is used to determine the threshold for successful fault detection with minimum false alarm and missed detection rate.

As physical and technological constraints arose in the industrial system due to complexity and robustness, many researchers developed modified and enhanced versions of the Kalman filter to cope with the advanced requirements of the system. As a result of dedicated research towards the development of stochastic fault detection techniques, in parallel with ongoing research on deterministic fault detection techniques, improved versions of Kalman filter is obtained, such as extended Kalman filters (EKF), unscented Kalman filters (UKF), adaptive Kalman filters, and augmented state Kalman filters.

An Extended Kalman filter is commonly used for estimating the non-measurable states of a nonlinear dynamic system (Jokic et al., 2018). Extended Kalman filter has shown good results random disturbances; however, owing to nonlinearity in states and measurements of the system, it is imperative to get the linearized and Jacobian matrix of the system model. Moreover, the linearization process reduces the estimation performance of EKF that might lead to instability of the filtering process for fault detection purposes.

Unscented Kalman filter overcomes the drawbacks of the EKF approach. UKF uses the unscented transform, i.e., a good approximation of the stochastic distribution of state rather than nonlinear function. Therefore, this method is straightforward and efficient for estimating the system states for nonlinear dynamic systems leading to better fault detection performance (Khazraj et al., 2016). However, in some cases, EKF requires a precise value of measurement and process noise covariance matrix. This condition is not often practical, leading to another version of the Kalman filter, i.e., adaptive Kalman filter.

An adaptive Kalman filter is used to tune the measurement noise covariance matrix and system noise covariance matrix according to noise conditions to obtain a satisfactory fault diagnosis (Hajiyev \& Soken, 2013). According to the literature, researchers classify the 
adaptive filters into four types: Bayesian-based, maximum likelihood-based, correlationbased, and covariance matching techniques (Tripathi et al., 2016). In addition, the augmented Kalman filter is often used to estimate the system states and fault and disturbance signals simultaneously (Gannouni \& Hmida, 2017). Various applications of Kalman filter-based fault detection system can be found in the gas turbine engine (Pourbabaee et al. 2016), synchronous generator (Nadarajan et al., 2016), power systems (Liu \& He, 2017), wind turbine system (Cho et al., 2018), and aircraft (Marzat et al., 2012).

\section{UIO-based Fault Detection}

Fault detection system should be robust against all undesired inputs such as process and measurement disturbance. Initially, it was proposed to decouple the unknown input from the state estimation process using the disturbance distribution matrix. If system states are decoupled from unknown disturbance, then residual is, obviously, also independent (Ding \& Frank, 1990; Wünnenberg \& Frank, 1987). The decoupling observer is known as the unknown input observer (UIO), a type of Luenberger observer, principally to estimate state variables.

In the Eigen structure assignment method, left eigenvectors of the observer gain matrix are assigned so that gain's left eigenvectors are orthogonal to the disturbance distribution matrix to make residual signal robust (Patton \& Chen, 2000). In this approach, instead of decoupling unknown input from the state estimation process, the residual signal is made independent of unknown input. The geometric approach is used by (Hur \& Ahn, 2014) to decouple the effect of disturbance from the residual. All the above approaches (UIO, Eigen structure assignment approach, and geometric approach) address the problem of disturbance decoupling from the residual. However, these approaches are not capable of handling the model uncertainties. One possible solution to tackle the model uncertainties is to model them as an unknown input and then apply the disturbance decoupling techniques further.

The inverter incipient sensor fault is successfully detected and accommodated in three-phase PWM inverters in the traction system (Zhang et al., 2017). UIO technique has been widely used in many applications of FD, aircraft systems (Hur \& Ahn, 2014), gas turbine engines (Dai et al., 2009), cyber-physical systems, and wind energy systems (Zhu $\&$ Gao, 2014). However, as stated in Ding (2013), the existing condition of the disturbance decoupling technique is stringent and hard to achieve for many practical systems. Moreover, the decoupling technique is also not suitable for cases where fault vector lies in the same space as disturbance vector that may lead to a decoupling of fault signal from residual as well, just like disturbance. An alternate strategy, widely adopted, is to design an observer to make a suitable compromise between robustness to unknown input and sensitivity to fault. It makes the fault detection problem a multi-objective design problem. 


\section{Optimized Observer/FDF-based Fault Detection}

Fault Detection Filter (FDF), a well-known realization of full order state observer, generates the residual for fault detection purposes. FDF structure for Equations 1 and 2 can be represented by the following Equation 9:

$$
\left\{\begin{array}{c}
\hat{x}(k+1)=A \hat{x}(k)+B u(k)+L(y(k)-\hat{y}(k)) \\
\hat{y}(k)=C \hat{x}(k)+D u(k) \\
r(k)=V(y(k)-\hat{y}(k)) \\
r(k)=V\left[C e(k)+F_{d} d(k)+F_{f} f(k)\right]
\end{array}\right.
$$

$\hat{x}(k) \in R^{n}, \hat{y}(k) \in R^{q}$ is the state estimation vector and measurement estimation vector, respectively. $r(k)$ is a so-called residual signal. $L$ and $V$ are two design parameters of FDF. Observer gain $L$ is determined in such a way that estimation error asymptotically goes to zero. The residual in Equation 9 can be rewritten in the frequency domain as Equation 10:

$$
r(z)=G_{r d} d(z)+G_{r f} f(z)
$$

Where

$$
\begin{aligned}
& G_{r d}=C(z I-A+L C)^{-1}\left(E_{d}-L F_{d}\right)+F_{d} \\
& G_{r f}=C(z I-A+L C)^{-1}\left(E_{f}-L F_{f}\right)+F_{f}
\end{aligned}
$$

It can be observed from Equation 9 that residual is dependent on fault and unknown input signal. $H_{\infty}$ norm represents the maximum influence of disturbance on residual and is widely used to improve the robustness of residual against the unknown inputs (Zhou \& Zhang, 2019). Furthermore, to analytically represent the influence of fault on the residual, $H_{\infty}$ norm, $H_{2}$ norm, and $H_{-}$index are successfully adopted for FDF design. Robustness to unknown input/disturbance while, at the same time, sensitivity to fault makes the design of FDF a multi-objective optimization problem. This way, optimal observer gain can be obtained by solving the following optimization problem over some specified frequency range (Equation 11).

$$
L^{*}, V^{*}=\arg \left(\max _{L, V}\left(\frac{\left\|G_{r f}\right\|}{\left\|G_{r d}\right\|}\right)\right)
$$

There are several forms of performance indices (i.e., $\frac{H_{\infty}}{H_{\infty}}, \frac{H_{-}}{H_{\infty}}, \frac{\mathrm{H}_{2}}{\mathrm{H}_{\infty}}$ ) are available for solving Equation 11. In addition, $L^{*}, V^{*}$ are optimal parameters of FDF in Equation 9 that deliver a residual which has maximum robustness to unknown inputs and maximum sensitivity to faults (Ding, 2013). 
Optimal FDF design for linear system formulated as a multi-objective optimization problem (Ding et al., 2000) and unified solution for the optimization problem is obtained using factorization technique which is realized by solving the Riccati equation. Aguilera et al. (2016) designed an observer-based fault detection system to detect the current sensor fault in the induction motor drive. A differential geometric approach is used in this study to detect and isolate the single and multiple faults, i.e., disconnection, offset, and constant gain faults.

The prime objective of all techniques developed for the solution of Equation 11 is to obtain an optimal trade-off between robustness and sensitivity. These techniques are well-suited for fault detection in linear systems subjected to unknown disturbances only. However, in model-based FD techniques, another issue often encounters in the residual generation process is model mismatching. A perfect mathematical model of a practical system is never available because of modeling error, process linearization, and component aging issues. Hence, optimization techniques that solve Equation 11 cannot be applied to uncertain systems.

System uncertainty severely affects the output estimation that leads to poor performance of fault detection. Robust FDF has been designed for continuous LTI systems subjected to disturbance and norm-bounded uncertainty (Zhong et al., 2003). Robust FD problem is formulated as $H_{\infty}$ model matching problem, and solution of the optimization problem is presented in linear matrix inequality (LMI) form in the said paper. Li et al. (2013) extended the same work discussed in Ding et al. (2000) for continuous-time linear uncertain systems subjected to polytopic uncertainty utilizing the iterative LMI approach. Farhat and Koenig (2015) formulated the proportional integral observer (PIO) design problem as a multi-objective optimization problem for the continuous-time linear uncertain system. The robustness to disturbance and uncertainty has been ensured minimizing the $H_{\infty}$ norm of $G_{r d}$ in the LMI framework.

Although the designed robust FDF, with and without system uncertainty, somehow minimizes the effect of disturbances and uncertainties, these are not completely decoupled from the residual. These unknown inputs still influence the residual. In such cases, the appropriate residual evaluation function and threshold computation selection are integral in successful fault detection. The final decision on the occurrence of a fault is made using a simple comparison between residual evaluation function and threshold.

There are two widely accepted ways to generate evaluation function and threshold depending on the system's dynamics under consideration. Generally, the norm-based residual evaluation function is used for a deterministic system in which the energy of unknown input is bounded under a certain limit $\left(\|d(k)\|_{2} \leq \delta_{d}\right)$. On the other hand, a statistical-based residual evaluation function is adopted for stochastic systems. For deterministic systems, $l_{2}$-norm is a mostly used evaluation function and is defined in [8]. Peak value, root mean square value, and moving average of residual is also used 
for residual evolution function. We refer our esteemed readers to Ding et al. (2003) for more knowledge and computational skills. In case, residual is dependent on disturbance, uncertainty, and fault signal, then residual, generated by any of the methods discussed above, can be represented as Equation 12:

$$
\|r(k)\|_{2}^{2}=\left\|r_{d}(k)+r_{u}(k)+r_{f}(k)\right\|_{2}^{2}
$$

In a fault-free case, $\left\|r_{f}(k)\right\|_{2}^{2}=0$, the threshold can be defined as Equation 13:

$$
J_{t h}=\sup \left(\|r(k)\|_{2}^{2}\right)
$$

\section{Other Observer-based Fault Detection Techniques}

Besides the previously discussed Kalman filter, UIO, and optimized FDF schemes, other types of fault detection observers in the literature have been investigated for LTI systems. Those are a proportional-integral observer (Do et al., 2018; Yang et al., 2020), sliding mode observer ( Zhirabok et al., 2018; Zhang et al., 2019), interval observer (Pourasghar et al., 2020; Zammali et al. 2020), and adaptive observer (Lijia et al., 2019; Perrin et al., 2004). These observers are designed to estimate the system output with minimum output estimation error and fast convergence speed. Then output estimation error is used as a residual to indicate the fault occurrence.

\section{DISCUSSION}

In this section, a comparison among various fault detection techniques discussed so far is given in terms of robustness, complexity, and performance. The robustness of the fault detection method is checked by the measure of sensitivity to noise, disturbance, and uncertainty. Likewise, robustness and the performance of the fault detection method are determined in terms of FAR and MDR. Based on the above survey, the following points are highlighted:

1. An observer generates the residual for fault detection, and it is synthesized to zero in fault-free cases. Observer-based and parity-space-based FD methods produce the alike residual in terms of residual characteristics. However, the observer-based method shows more robustness to uncertainty as compared to the parity-spacebased residual generator.

2. FDF is a complete state observer, while an unknown input observer is a reducedorder observer and might be considered where full state estimation is not required. UIO has more robustness to unknown inputs with increased complexity and computational effort.

3. Observer-based and parity-space-based fault detection methods are designed for additive faults and perform well when the plant model is perfect. 
4. Observer-based and parity-space-based FD system design requires the knowledge of robust control theory when there is uncertainty and unmodeled disturbance in the linear systems. These unwanted inputs are handled by selecting a higher detection threshold in the second step of the fault detection process. However, a higher detection threshold causes to increase in the FAR, and a lower detection threshold cause to increase in the MDR. Therefore, a feasible trade-off is required between FAR and MDR for successful fault detection.

5. Optimization-based FD methods are formulated so that the sensitivity of residual to unknown inputs is minimized along with improved sensitivity to faults. Such methods provide a solution in terms of mathematical multi-objective functions. There could be one disadvantage: it might not guarantee the usefulness and performance of the solution in some applications. Hence, special care is required while implementing optimized FD methods in the underlying application, which may also increase complexity.

6. Kalman filter-based FD system is used for stochastic systems, and much knowledge of statistical analysis and probability is required. Different versions of the Kalman filter can be applied to nonlinear systems and time-varying systems as well.

7. Fault detection methods relying on system identification are useful for linear and nonlinear systems, but the performance of such FD systems entirely depends on detecting the variation in system parameters. Moreover, these fault detection methods are beneficial for detecting small and incipient faults.

8. The major disadvantage of the model-based FD method is to get the precise mathematical model of the system. This reason restricts the application of the model-based FD methods to industrial systems. In such cases, data-driven techniques are advantageous when the system model is unavailable (Denkena et al., 2020).

9. The parameter-estimation method produced good results in detecting structural damage, while all other model-based methods are well suited for detecting the actuator and sensor faults.

10. The selection of an appropriate detection technique depends on the reliability of the available knowledge of the system.

\section{COMPARISON OF FAULT DETECTION TECHNIQUES}

This section compares two well-known fault detection techniques in terms of detectability, design complexity, and computational load. For this purpose, a linear DC motor model is simulated in MATLAB for sensor and actuator fault detection. First, a unified solution of an optimal FDF developed in Ding (2013) for Equations 1 and 2 subjected to deterministic disturbance is compared with Kalman filter-based optimal estimator for stochastic LTI 
system (Blanke et al., 2015). Both FD techniques confirm good performance in terms of fault detection if an unknown disturbance lies within bound and there is no system uncertainty. Second, the state-space model of DC motor in Equations 1 and 2 has the following matrices:

$$
\begin{aligned}
& A=[0.22310 .0109 ;-0.00220 .5488], B=[0.0047 ; 0.4512], C=\operatorname{eye}(2), D=0 \\
& E_{d}=E_{\omega}=[-1.5537 ; 0], F_{d}=F_{f}=C, E_{f}=B
\end{aligned}
$$

\section{Kalman Filter Design}

Residual generation for stochastic LTI system (Equation 14):

$$
\begin{aligned}
& x(k+1)=A x(k)+B u(k)+E_{\omega} \omega(k)+E_{f} f(k) \\
& y(k)=C x(k)+D u(k)+v(k)+F_{f} f(k)
\end{aligned}
$$

Let $\omega(k)$ and $v(k)$, be zero-mean process and measurement noise with following covariance matrix, $E\left(\left[\begin{array}{c}\omega(k) \\ v(k)\end{array}\right]\left[\begin{array}{cc}\omega^{T}(i) & v^{T}(i)\end{array}\right]\right)=\left[\begin{array}{cc}\sigma^{2} \omega & S_{k i} \\ S_{i k} & \sigma^{2}{ }_{v}\end{array}\right]$. The dynamics of the Kalman filter-based residual generator is represented by Equation 15:

$$
\begin{aligned}
& \hat{x}(k+1)=A \hat{x}(k)+B u(k)+K(y(k)-\hat{y}(k)) \\
& r(k)=y(k)-\hat{y}(k)=C e(k)+v(k)+F_{f} f(k)
\end{aligned}
$$

$K$ is Kalman filter gain and can be determined as $K=\left(A P C^{T}+E_{d} S\right)\left[C P C^{T}+\sigma^{2}{ }_{v}\right]^{-1}$, While $P$ can be obtained by solving the following Riccati Equation 16:

$$
P=A P A^{T}-K\left[C P C^{T}+{\sigma_{v}}^{2}\right] K^{T}+\sigma^{2}{ }_{\omega}
$$

The residual evaluation and threshold are given as Equation 17:

$$
J(k)=r(k)^{T}\left(\sigma_{R E S(k)}^{2}\right)^{-1} r(k)
$$

Where $\sigma_{R E S}^{2}=E\left[r(k) r^{T}(k)\right]=C P C^{T}+\sigma^{2}{ }_{v}$. Threshold could be defined as $J_{t h}=\chi_{\alpha}^{2}(n)$, where $\alpha$ is given false alarm rate (FAR) and $n$ is the number of output signals. The threshold is computed using chi-square $\left(X^{2}\right)$ test.

\section{Unified Solution of Optimal FDF}

Residual generation for deterministic LTI system in Equations 1 and $2, d(k)$ is $l_{2}$ norm bounded unknown disturbance, holds $\left(\|d(k)\|_{2} \leq \delta_{d}\right)$. Optimal FDF of the form in Equation 11, observer gain $L$ and post-filter $V$ is obtained using the unified solution 
Equation 18:

$$
\begin{aligned}
& L=\left(A X C^{T}+E_{d} F_{d}^{T}\right)\left(F_{d} F_{d}{ }^{T}+C X C^{T}\right)^{-1} \\
& V=\left(F_{d} F_{d}{ }^{T}+C X C^{T}\right)^{-0.5} \\
& X=A X A^{T}-L\left(F_{d} F_{d}^{T}+C X C^{T}\right) L^{T}+E_{d} E_{d}^{T}
\end{aligned}
$$

Where, $X$ is a solution of the above Riccati equation. Residual obtained from unified solution is an optimal solution because, in fault-free case, the effect of $d(k)$ on the residual is uniform in the entire subspace spanned by the disturbance $d(k)$. Thus Equation 9 serves as a residual evaluation function, and the threshold is taken as the upper limit of disturbance energy (Equation 19).

$$
J_{t h}=\delta_{d}
$$

Table 1 describes the comparison in gain matrices of the Kalman filter and optimal FDF. It is shown that the gain of both filters depends on their noise characteristics. The following comparison reveals no difference between these two filters, and they deliver optimal residual

\begin{tabular}{|c|c|}
\hline$K=\left(A P C^{T}+E_{d} S\right)\left[C P C^{T}+\sigma_{v}^{2}\right]^{-1}$ & $L=\left(A X C^{T}+E_{d} F_{d}^{T}\right)\left(F_{d} F_{d}^{T}+C X C^{T}\right)^{-1}$ \\
\hline$P=A P A^{T}-K\left[C P C^{T}+\sigma_{v}^{2}\right] K^{T}+\sigma_{\omega}^{2}$ & $X=A X A^{T}-L\left(F_{d} F_{d}^{T}+C X C^{T}\right) L^{T}+E_{d} E_{d}^{T}$ \\
\hline$J(k)=r(k)^{T}\left(\sigma_{R E S(k)}^{2}\right)^{-1} r(k)$ & $J(k)=r^{T}(k) V^{T} \operatorname{Vr}(k)$ \\
\hline$\sigma^{2}{ }_{R E S}=C P C^{T}+\sigma_{v}^{2}$ & $V=\left(F_{d} F_{d}^{T}+C X C^{T}\right)^{-0.5}$ \\
\hline$E_{d} S$ & $E_{d} F_{d}^{T}$ \\
\hline$\sigma_{v}^{2}$ & $F_{d} F_{d}^{T}$ \\
\hline$\sigma_{\omega}^{2}$ & $E_{d} E_{d}^{T}$ \\
\hline
\end{tabular}
in terms of robustness if there is no system uncertainty.

Table 1

Comparison between FD techniques via gain equations

$\mathbf{r}(\mathbf{k})$ is zero-mean white noise $\quad \mathrm{r}(\mathrm{k})$ is an all-pass filter in fault-free case, with the covariance matrix $\sigma^{2} \mathbf{v} \quad$ i.e., the beauty of a unified solution

\section{Comparison by Simulation}

For simulation purposes, the following assumptions are considered, $x(0)=[0,0]^{T}$, $u(k)=0.5$, the covariance of Gaussiannoise: $\sigma_{v}^{2}=0.0025, \sigma^{2}{ }_{\omega}=0.0012$, FAR $=10 \%$ and $d(k) \in[-0.01,0.01]$. 
The following simulation results have been observed: Figure 2 illustrates that system output estimates are close to actual measurement in fault-free cases. Thus, residual obtained from both schemes prove robustness to unknown disturbance/noise. Figure 3 demonstrates a speed sensor fault case in which ramp input as an incipient fault is injected at $2.2 \mathrm{sec}$. It is observed that the detection time of the Kalman filter is high as compared to optimal FDF. In this case, the Kalman filter has shown less robustness to a time-varying fault in the DC motor speed sensor. Finally, Figure 4 confirms the effectiveness of both schemes in terms of robustness and detection time in actuator stuck fault conditions.

Simulation results confirm that both approaches have shown better performance under unknown inputs. However, they have limited capability of FD in the presence of system uncertainty and may deliver large FAR and MDR. Therefore, a fault-sensitive filter has

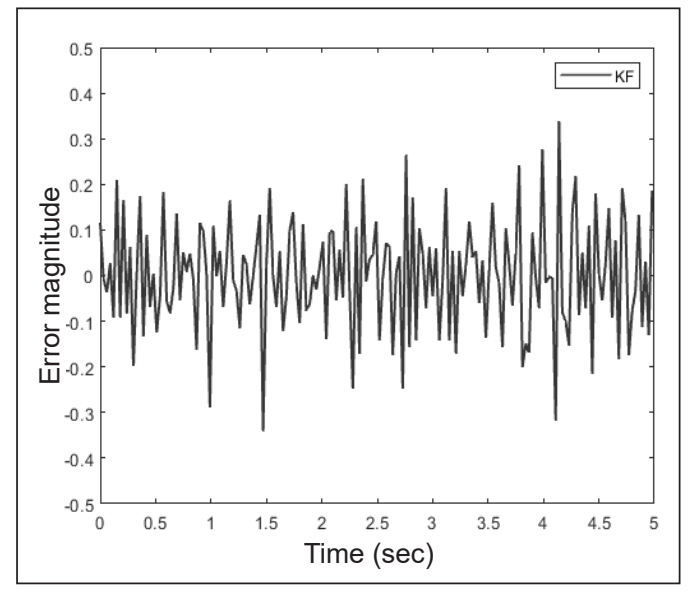

(a)

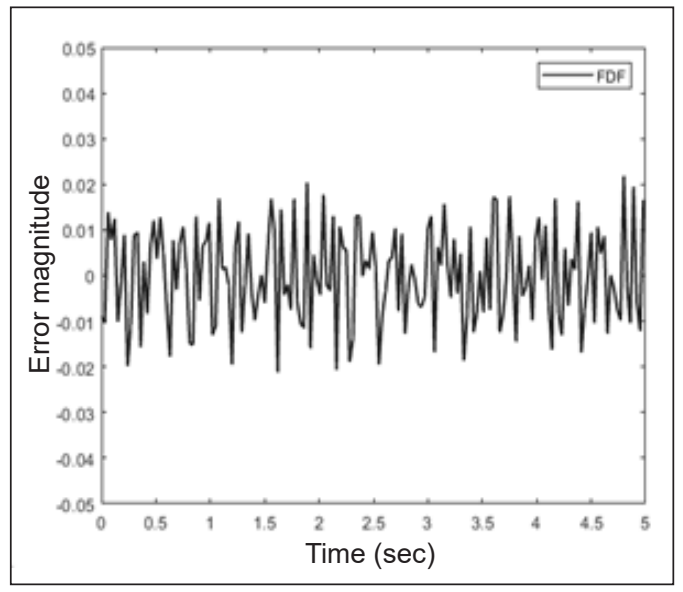

(b)

Figure 2. Residual, $\mathrm{f}(\mathrm{k})=0$ from (a) Kalman filter (left); and (b) optimal FDF

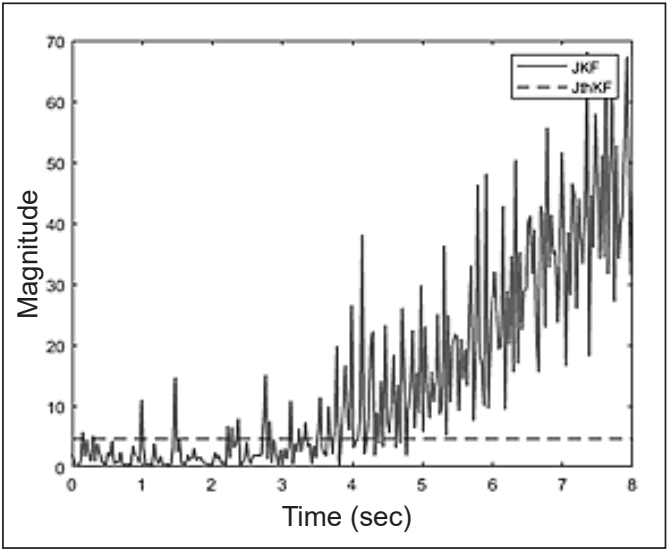

(a)

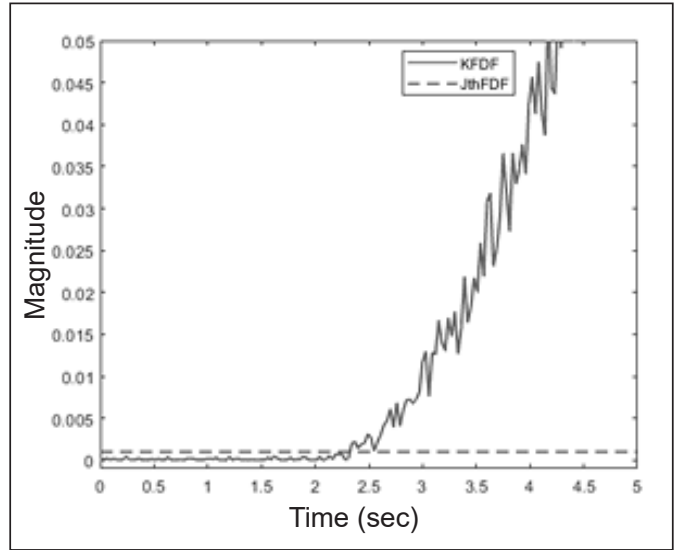

(b)

Figure 3. Evaluation function and threshold in incipient fault at $\mathrm{t}=2.2 \mathrm{sec}$ : (a) Kalman filter; and (b) optimal FDF 


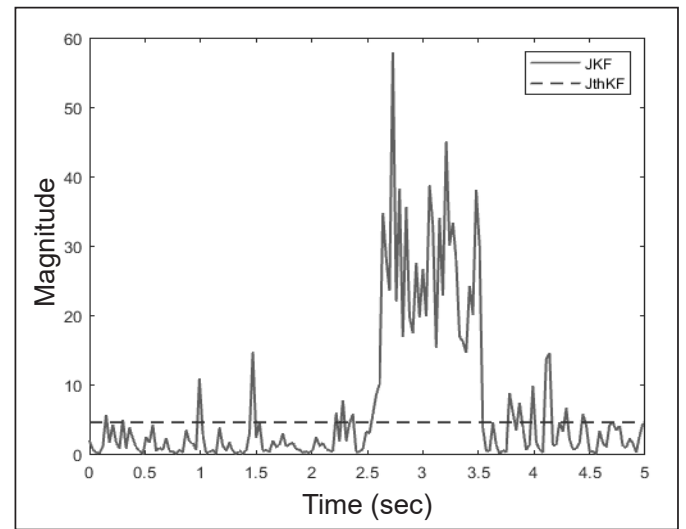

(a)

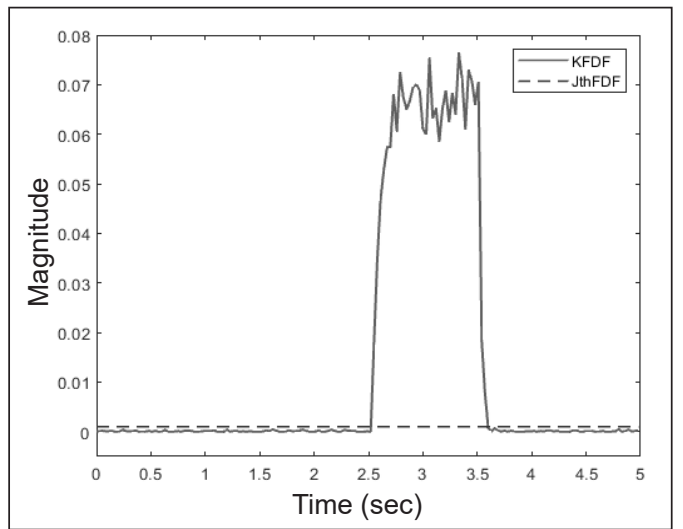

(b)

Figure 4. Evaluation function and threshold in actuator stuck fault at $\mathrm{t}=2.5-3.5 \mathrm{sec}$ : (a) Kalman filter; and (b) optimal FDF

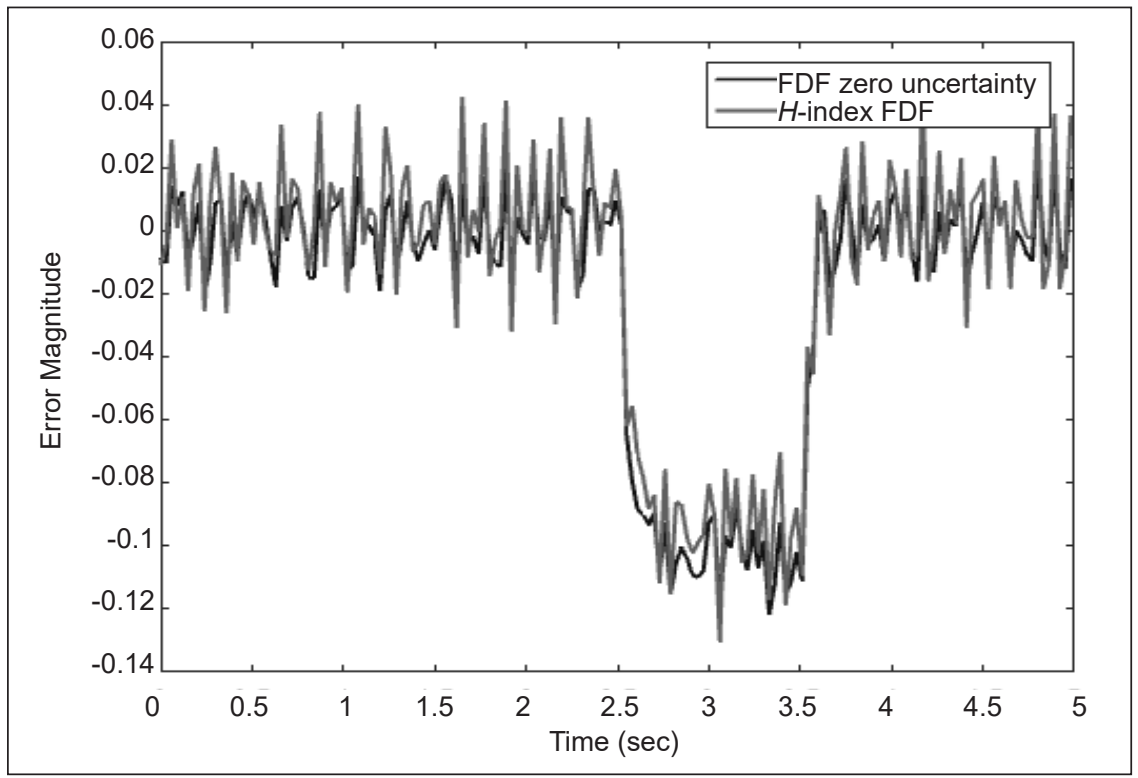

Figure 5. Residual in actuator stuck case with system uncertainty

been designed for LTI uncertain system to handle the uncertainty problem (Ahmad \& Mohd-Mokhtar, 2020). Figure 5 shows residual obtained from $H$-index FDF for uncertain DC motor system and residual from optimal FDF of nominal DC motor system. It has been shown that $H$-index fault-sensitive FDF can minimize the effect of system uncertainties and enhance the fault sensitivity (Ahmad \& Mohd-Mokhtar, 2020) and shows the approximate performance of optimal FDF designed for nominal DC motor. Detail comparison of the three FD schemes is given in Table 2. 
Table 2

General comparison of three FD schemes

\begin{tabular}{|c|c|c|c|}
\hline & Kalman Filter & Optimal FDF & $H$-index FDF \\
\hline Detectability & $\begin{array}{l}\text { Can detect all faults but may } \\
\text { increase FAR when the fault has } \\
\text { a low fault to noise ratio }\end{array}$ & $\begin{array}{l}\text { Can detect all faults } \\
\text { simulated in this paper }\end{array}$ & $\begin{array}{l}\text { Can detect all faults } \\
\text { simulated in this paper }\end{array}$ \\
\hline Detection time & Fast & Fast & Fast \\
\hline Computation load & Moderate & Low & Moderate \\
\hline $\begin{array}{l}\text { Robustness to } \\
\text { uncertainty }\end{array}$ & FAR high in the simulation case & $\begin{array}{l}\text { MDR high in the } \\
\text { simulation case }\end{array}$ & Acceptable robustness \\
\hline $\begin{array}{l}\text { Robustness to initial } \\
\text { states }\end{array}$ & Less robust than optimal FDF & More robust than other & Least robust \\
\hline
\end{tabular}

\section{MODERN TRENDS IN MODEL-BASED FD FOR LTI SYSTEMS}

1. As discussed above, a model-based FD system consists of two stages: residual generation and residual evaluation. The prime objective of the residual generation stage is to generate the optimal residual. The objective of the residual evaluation and threshold stage is to ensure maximum fault detectability. Thus, the overall objective of an optimal fault detection system is to achieve the maximum fault detectability and maintain zero false alarm for a deterministic type of system or achieve the fault detectability under some allowable false alarm rate under stochastic noise. Based on the survey, most of the results mentioned above focus on the effort of residual generation. However, little attention is given to the residual evaluation and corresponding threshold computation. Hence, there is an indispensable need to design the residual generation and residual evaluation in an integrated way, rather than dealing with them separately, to optimize certain criteria for better performance of the fault detection system.

2. Even though very nice results are available for model-based fault detection, there is still a scarcity of research for uncertain linear systems. The standalone design of the residual generator and residual evaluator and an optimally integrated fault detection system, as discussed in 1, for the continuous and discrete-time linear uncertain system, is still an open and challenging topic for researchers.

3. The fault should be estimated after it has been detected so that its effect can be compensated to maintain the reliability of a practical system in a faulty situation. An optimal fault estimator and fault-tolerant controller for nominal and uncertain systems is also an ongoing open research topic.

4. It is also observed that minimal effort has been devoted to developing fault detection systems in a closed-loop environment. Open-loop fault detection techniques become ineffective in a closed-loop environment. Thus, it is necessary to work on this topic which is also an emerging topic. 
5. Besides the process and measurement noise, there is also multiplicative noise in the system. Due to this, traditional methods could not produce the optimal performance for fault detection. Hence, there is a need to address the fault detection problem for LTI systems involving multiplicative noise.

In some instances, recorded or online data of system inputs and measurements are only available. In such scenarios, model-based fault detection methods cannot be directly applied. Therefore, model-based and data-driven or signal processing-based fault detection methods can be combined to design optimal FD systems for complex systems where the mathematical model of the system is not possible. As technology advances and more techniques are developed regarding fault detection in LTI systems, the combination of data-driven and model-based fault detection methods are the future research directions. The integration of the two methods provides new opportunities and challenges. Furthermore, the integration of robust control and machine learning techniques for observer-based FD and estimation scheme for linear systems open new research directions in the model-based FD framework.

\section{CONCLUSION}

The safety, reliability and desired performance of a practical system must be maintained at all the time during its operation. Fault detection plays a paramount role in accomplishing these objectives. This paper discusses various model-based residual generation techniques, including FDF, UIO, parity-space, optimization-based, Kalman filter, and system identification approach. Three different FD approaches were illustrated to address the FD problem in the DC motor system, and their performance is compared. The arising issues and emerging research topics on fault detection for LTI systems were explained. The outcome from this paper may assist in further research in the future.

\section{ACKNOWLEDGMENT}

The work is supported under the HRDI-UESTPS scholarship program funded by the Higher Education Commission (HEC) of Pakistan and partially supported by the USM RUI Grant: 1001/PELECT/8014093.

\section{REFERENCES}

Aguilera, F., de la Barrera, P. M., De Angelo, C. H., \& Espinoza Trejo, D. R. (2016). Current-sensor fault detection and isolation for induction-motor drives using a geometric approach. Control Engineering Practice, 53, 35-46. https://doi.org/10.1016/j.conengprac.2016.04.014

Ahmad, M., \& Mohd-Mokhtar, R. (2020). H-indexed fault sensitive filter design for linear discrete-time uncertain DC motor system. Interciencia, 45(10), 60-74. 
Ahmad, S., Ali, N., Ayaz, M., \& Ahmad, E. (2017). Design of robust fault detection filter using algorithm for a class of LTI systems. In 13th International Conference on Emerging Technologies (ICET) (pp. 1-5). IEEE Publishing. https://doi.org/10.1109/ICET.2017.8281720

Bachir, S., Tnani, S., Trigeassou, J. C., \& Champenois, G. (2006). Diagnosis by parameter estimation of stator and rotor faults occurring in induction machines. IEEE Transactions on Industrial Electronics, 53(3), 963-973. https://doi.org/10.1109/TIE.2006.874258

Belmokhtar, K., Ibrahim, H., \& Merabet, A. (2015). Online parameter identification for a DFIG driven wind turbine generator based on recursive least squares algorithm. In IEEE 28th Canadian Conference on Electrical and Computer Engineering (CCECE) (pp. 965-969). IEEE Publishing. https://doi.org/10.1109/ CCECE.2015.7129406

Blanke, M., Kinnaert, M., Lunze, J., \& Staroswiecki, M. (2015). Diagnosis and fault-tolerant control (3rd Ed.). Springer.

Bøgh, S. (1995). Multiple hypothesis-testing approaches to FDI for the industrial actuator benchmark. Control Engineering Practice, 3(12), 1763-1768. https://doi.org/10.1016/0967-0661(95)00191-V

Chen, W., Ding, S. X., Haghani, A., Naik, A., Khan, A. Q., \& Yin, S. (2011). Observer-based FDI schemes for wind turbine benchmark. IFAC Proceedings Volumes, 44(1), 7073-7078. https://doi.org/10.3182/201108286-IT-1002.03469

Cho, S., Gao, Z., \& Moan, T. (2018). Model-based fault detection, fault isolation, and fault-tolerant control of a blade pitch system in floating wind turbines. Renewable Energy, 120, 306-321. https://doi.org/10.1016/j. renene.2017.12.102

Da, R., \& Lin, C. F. (1996). Sensitivity analysis of the state chi-square test. IFAC Proceedings Volumes, 29(1), 6596-6601. https://doi.org/10.1016/S1474-6670(17)58741-8

Dai, X., Gao, Z., Breikin, T., \& Wang, H. (2009). Disturbance attenuation in fault detection of gas turbine engines: A discrete robust observer design. IEEE Transactions on Systems, Man, and Cybernetics, Part C (Applications and Reviews), 39(2), 234-239. https://doi.org/10.1109/TSMCC.2008.2005845

Denkena, B., Bergmann, B., \& Stoppel, D. (2020). Reconstruction of process forces in a five-axis milling center with a LSTM neural network in comparison to a model-based approach. Journal of Manufacturing and Materials Processing, 4(3), Article 62. https://doi.org/10.3390/jmmp4030062

Ding, S. X. (2013). Model-based fault diagnosis techniques: design schemes, algorithms, and tools. Springer Science \& Business Media.

Ding, S. X. (2014). Data-driven design of fault diagnosis and fault-tolerant control systems. Springer. https:// doi.org/10.1007/978-1-4471-6410-4

Ding, S. X., \& Frank, P. M. (1990). Fault detection via factorization approach. Systems \& Control Letters, 14(5), 431-436. https://doi.org/10.1016/0167-6911(90)90094-B

Ding, S. X., Jeinsch, T., Frank, P. M., \& Ding, E. L. (2000). A unified approach to the optimization of fault detection systems. International Journal of Adaptive Control and Signal Processing, 14(7), 725-745. https://doi.org/10.1002/1099-1115(200011) 
Ding, S. X., Zhang, P., \& Frank, P. M. (2003). Threshold calculation using LMI-technique and its integration in the design of fault detection systems. In 42nd IEEE International Conference on Decision and Control (pp. 469-474). IEEE Publishing. https://doi.org/10.1109/CDC.2003.1272607

Do, M. H., Koenig, D., \& Theilliol, D. (2018). Robust $\mathrm{H} \infty$ proportional-integral observer for fault diagnosis: Application to vehicle suspension. IFAC-PapersOnLine, 51(24), 536-543. https://doi.org/10.1016/j. ifacol.2018.09.628

Doraiswami, R., \& Cheded, L. (2013). A unified approach to detection and isolation of parametric faults using a Kalman filter residual-based approach. Journal of the Franklin Institute, 350(5), 938-965. https://doi. org/10.1016/j.jfranklin.2013.01.005

Dybkowski, M., \& Klimkowski, K. (2017). Speed sensor fault detection algorithm for vector control methods based on the parity relations. In 2017 19th European Conference on Power Electronics and Applications (pp. 1-5). IEEE Publishing. https://doi.org/10.23919/EPE17ECCEEurope.2017.8099342

Farhat, A., \& Koenig, D. (2015). PI robust fault detection observer for a class of uncertain switched systems using LMIs. IFAC-PapersOnLine, 48(21), 125-130. https://doi.org/10.1016/j.ifacol.2015.09.515

Frank, P. M., Ding, S. X., \& Koppen-Seliger B. (2000). Current developments in the theory of FDI. IFAC Proceeding Volumes: 4th IFAC Symposium on Fault Detection, Supervision, and Safety for Technical Processes, 33(11), 17-28.

Franklin, G. F., David-Powell, J., \& Emami-Naeini, A. (2019). Feedback control of dynamic systems (8th Ed.). Pearson Prentice Hall.

Gannouni, F., \& Hmida, F. B. (2017). Simultaneous state and fault estimation for linear stochastic systems. In 18th International Conference on Sciences and Techniques of Automatic Control and Computer Engineering (STA) (pp. 59-66). IEEE Publishing. https://doi.org/10.1109/STA.2017.8314965

Gao, Z., Cecati, C., \& Ding, S. X. (2015). A survey of fault diagnosis and fault-tolerant techniques-part I: Fault diagnosis with model-based and signal-based approaches. IEEE Transactions on Industrial Electronics, 62(6), 3757-3767. https://doi.org/10.1109/TIE.2015.2417501

Gautam, S., Tamboli, P. K., Patankar, V. H., Duttagupta, S. P., \& Roy, K. (2017). Performance evaluation of statistical method for incipient fault detection under noisy environment. IFAC-PapersOnLine, 50(1), 15728-15733. https://doi.org/10.1016/j.ifacol.2017.08.2415

Gertler, J. J. (2017). Fault detection and diagnosis in engineering systems. CRC Press. https://doi. org/10.1201/9780203756126

Hajiyev, C., \& Soken, H. E. (2013). Robust adaptive Kalman filter for estimation of UAV dynamics in the presence of sensor/actuator faults. Aerospace Science and Technology, 28(1), 376-383. https://doi. org/10.1016/j.ast.2012.12.003

Herrera, L., \& Yao, X. (2018). Parameter identification approach to series DC arc fault detection and localization. In IEEE Energy Conversion Congress and Exposition (ECCE) (pp. 497-501). IEEE Publishing. https:// doi.org/10.1109/ECCE.2018.8557679

Hur, H., \& Ahn, H. S. (2014). Unknown input H-infinity observer-based localization of a mobile robot with sensor failure. IEEE/ASME Transactions on Mechatronics, 19(6), 1830-1838. https://doi.org/10.1109/ TMECH.2014.2298034 
Hwang, W., \& Huh, K. (2015). Fault detection and estimation for electromechanical brake systems using parity space approach. Journal of Dynamic Systems, Measurement, and Control, 137(1), Article 014504. https://doi.org/10.1115/1.4028184

Isermann, R. (1984). Process fault detection based on modeling and estimation methods - A survey. Automatica, 20(4), 387-404. https://doi.org/10.1016/0005-1098(84)90098-0

Isermann, R. (1997). Supervision, fault-detection, and fault-diagnosis methods - An introduction. Control Engineering Practice, 5(5), 639-652. https://doi.org/10.1016/S0967-0661(97)00046-4

Isermann, R. (2006). Fault-diagnosis systems. Springer.

Jesica, E., \& Poznyak, A. (2018). Parameter estimation in continuous-time stochastic systems with correlated noises using the Kalman filter and least squares method. IFAC-PapersOnLine, 51(13), 309-313. https:// doi.org/10.1016/j.ifacol.2018.07.296

Jie, C., \& Patton, R. J. (2012). Robust model-based fault diagnosis for dynamic systems. Springer. https://doi. org/10.1007/978-1-4615-5149-2

Jokic, I., Zecevic, Z., \& Krstajic, B. (2018). State-of-charge estimation of lithium-ion batteries using extended Kalman filter and unscented Kalman filter. In 23rd International Scientific-Professional Conference on Information Technology (IT) (pp. 1-4). IEEE Publishing. https://doi.org/10.1109/SPIT.2018.8350462

Khang, H. V., Kandukuri, S., Pawlus, W., \& Robbersmyr, K. G. (2018). Parameter identification of a winding function-based model for fault detection of induction machines. In Eighth International Conference on Information Science and Technology (ICIST) (pp. 200-205). https://doi.org/10.1109/ICIST.2018.8426188

Khazraj, H., Faria da Silva, F., \& Bak, C. L. (2016). A performance comparison between extended Kalman filter and unscented Kalman filter in power system dynamic state estimation. In 51st International Universities Power Engineering Conference (UPEC) (pp. 1-6). IEEE Publishing. https://doi.org/10.1109/ UPEC.2016.8114125

Kleilat, I., Al-Sheikh, H., Moubayed, N., \& Hoblos, G. (2018). Robust fault diagnosis of sensor faults in power converter used in hybrid electric vehicle. IFAC-PapersOnLine, 51(24), 326-331. https://doi.org/10.1016/j. ifacol.2018.09.597

Li, L., Ding, S. X., Zhang, Y., \& Yang, Y. (2016). Optimal fault detection design via iterative estimation methods for industrial control systems. Journal of the Franklin Institute, 353(2), 359-377. https://doi. org/10.1016/j.jfranklin.2015.12.002

Li, W., Zhu, Z., Zhou, G., \& Chen, G. (2013). Optimal H i /H $\infty$ fault-detection filter design for uncertain linear time-invariant systems: An iterative linear matrix inequality approach. IET Control Theory \& Applications, 7(8), 1160-1167. https://doi.org/10.1049/iet-cta.2012.0954

Lijia, C., Yu, T., \& Guo, Z. (2019). Adaptive observer-based fault detection and active tolerant control for unmanned aerial vehicles attitude system. IFAC-PapersOnLine, 52(24), 47-52. https://doi.org/10.1016/j. ifacol.2019.12.379

Liu, X., Wang, Z., Wang, Y., \& Shen, Y. (2018). Dynamic threshold computation in fault detection for discretetime linear systems. In 2018 Chinese Control And Decision Conference (CCDC) (pp. 2241-2246). IEEE Publishing. https://doi.org/10.1109/CCDC.2018.8407499 
Liu, Z., \& He, H. (2017). Sensor fault detection and isolation for a lithium-ion battery pack in electric vehicles using adaptive extended Kalman filter. Applied Energy, 185, 2033-2044. https://doi.org/10.1016/j. apenergy.2015.10.168

Marzat, J., Piet-Lahanier, H., Damongeot, F., \& Walter, E. (2012). Model-based fault diagnosis for aerospace systems: A survey. Proceedings of the Institution of Mechanical Engineers, Part G: Journal of Aerospace Engineering, 226(10), 1329-1360. https://doi.org/10.1177/0954410011421717

Mehra, R. K., \& Peschon, J. (1971). An innovations approach to fault detection and diagnosis in dynamic systems. Automatica, 7(5), 637-640. https://doi.org/10.1016/0005-1098(71)90028-8

Na, Y., \& Ahmad, M. (2019). A fault detection scheme for switched systems with noise under asynchronous switching. In 9th International Conference on Information Science and Technology (ICIST) (pp. 258-262). IEEE Publishing. https://doi.org/10.1109/ICIST.2019.8836838

Nadarajan, S., Panda, S. K., Bhangu, B., \& Gupta, A. K. (2016). Online model-based condition monitoring for brushless wound-field synchronous generator to detect and diagnose stator windings turn-to-turn shorts using extended Kalman filter. IEEE Transactions on Industrial Electronics, 63(5), 3228-3241. https:// doi.org/10.1109/TIE.2016.2535959

Nikiforov, I., Varavva, V., \& Kireichikov, V. (1993). Application of statistical fault detection algorithms to navigation systems monitoring. Automatica, 29(5), 1275-1290. https://doi.org/10.1016/00051098(93)90050-4

Odendaal, H. M., \& Jones, T. (2014). Actuator fault detection and isolation: An optimized parity space approach. Control Engineering Practice, 26, 222-232. https://doi.org/10.1016/j.conengprac.2014.01.013

Patton, R. J., \& Chen, J. (2000). On eigenstructure assignment for robust fault diagnosis. International Journal of Robust and Nonlinear Control, 10(14), 1193-1208. https://doi.org/10.1002/10991239(20001215)10:14<1193::AID-RNC523>3.0.CO;2-R

Perrin, O., Basseville, M., Sorine, M., \& Zhang, Q. (2004). On-board diesel particulate filter fault detection using an adaptive observer. IFAC Proceedings Volumes, 37(22), 367-372. https://doi.org/10.1016/S1474$6670(17) 30371-3$

Pourasghar, M., Puig, V., \& Ocampo-Martinez, C. (2020). Characterization of interval-observer fault detection and isolation properties using the set-invariance approach. Journal of the Franklin Institute, 357(3), 18531886. https://doi.org/10.1016/j.jfranklin.2019.11.027

Pourbabaee, B., Meskin, N., \& Khorasani, K. (2016). Sensor fault detection, isolation, and identification using multiple-model-based hybrid Kalman filter for gas turbine engines. IEEE Transactions on Control Systems Technology, 24(4), 1184-1200. https://doi.org/10.1109/TCST.2015.2480003

Rasoolzadeh, A., \& Salmasi, F. R. (2020). Mitigating zero dynamic attacks in communication link-enabled droop-controlled hybrid AC/DC microgrids. IET Cyber-Physical Systems: Theory \& Applications, 5(2), 207-217. https://doi.org/10.1049/iet-cps.2019.0043

Sun, B., Wang, J., He, Z., Qin, Y., Wang, D., \& Zhou, H. (2019). Fault detection for closed-loop control systems based on parity space transformation. IEEE Access, 7, 75153-75165. https://doi.org/10.1109/ ACCESS.2019.2916785 
Tripathi, R. P., Ghosh, S., \& Chandle, J. O. (2016). Tracking of object using optimal adaptive Kalman filter. In 2016 IEEE International Conference on Engineering and Technology (ICETECH) (pp. 1128-1131). IEEE Publishing. https://doi.org/10.1109/ICETECH.2016.7569426

Wang, Y., Liu, Q., Li, K., Yin, L., \& Chen, H. (2019). Resilient fault and attack detection of DCT vehicles using parity space approach. In 2019 Chinese Automation Congress (CAC) (pp. 431-436). IEEE Publishing. https://doi.org/10.1109/CAC48633.2019.8996359

Willsky, A., \& Jones, H. (1976). A generalized likelihood ratio approach to the detection and estimation of jumps in linear systems. IEEE Transactions on Automatic Control, 21(1), 108-112. https://doi.org/10.1109/ TAC.1976.1101146

Wünnenberg, J., \& Frank, P. M. (1987). Sensor fault detection via robust observers. In S. Tzafestas, M. Singh \& G. Schmidt (Eds.), System Fault Diagnostics, Reliability and Related Knowledge-Based Approaches (pp. 147-160). Springer. https://doi.org/10.1007/978-94-009-3929-5_5

Yang, X., Chen, Y., Li, B., \& Luo, D. (2020). Battery states online estimation based on exponential decay particle swarm optimization and proportional-integral observer with a hybrid battery model. Energy, 191, Article 116509. https://doi.org/10.1016/j.energy.2019.116509

Ye, H., Wang, W., \& Zhai, S. (2015). Fault diagnosis based on parameter estimation in closed-loop systems. IET Control Theory \& Applications, 9(7), 1146-1153. https://doi.org/10.1049/iet-cta.2014.0717

Zammali, C., Van Gorp, J., Wang, Z., \& Raïssi, T. (2020). Sensor fault detection for switched systems using interval observer with Lo performance. European Journal of Control, 57, 147-156. https://doi. org/10.1016/j.ejcon.2020.06.004

Zhang, K., Jiang, B., Yan, X. G., \& Mao, Z. (2017). Incipient sensor fault estimation and accommodation for inverter devices in electric railway traction systems. International Journal of Adaptive Control and Signal Processing, 31(5), 785-804. https://doi.org/10.1002/acs.2730

Zhang, P., Ye, H., Ding, S. X., Wang, G. Z., \& Zhou, D. H. (2006). On the relationship between parity space and approaches to fault detection. Systems \& Control Letters, 55(2), 94-100. https://doi.org/10.1016/j. sysconle.2005.05.006

Zhang, Y., \& Jiang, J. (2008). Bibliographical review on reconfigurable fault-tolerant control systems. Annual Reviews in Control, 32(2), 229-252. https://doi.org/10.1016/j.arcontrol.2008.03.008

Zhang, Z. H., Li, S., Yan, H., \& Fan, Q. Y. (2019). Sliding mode switching observer-based actuator fault detection and isolation for a class of uncertain systems. Nonlinear Analysis: Hybrid Systems, 33, 322-335. https://doi.org/10.1016/j.nahs.2019.04.001

Zhirabok, A. N., Shumsky, A. E., \& Zuev, A. V. (2018). Sliding mode observers for fault detection in linear dynamic systems. IFAC-PapersOnLine, 51(24), 1403-1408. https://doi.org/10.1016/j.ifacol.2018.09.540

Zhong, M., Ding, S. X., Lam, J., \& Wang, H. (2003). An LMI approach to design robust fault detection filters for uncertain LTI systems. Automatica, 39(3), 543-550. https://doi.org/10.1016/S0005-1098(02)00269-8

Zhong, M., Song, Y., Xue, T., Yang, R., \& Li, W. (2018). Parity space-based fault detection by minimum error minimax probability machine. IFAC-PapersOnLine, 51(24), 1292-1297. https://doi.org/10.1016/j. ifacol.2018.09.568 
Masood Ahmad and Rosmiwati Mohd-Mokhtar

Zhou, J., \& Zhang, D. (2019). H-infinity fault detection for delta operator systems with random two-channels packet losses and limited communication. IEEE Access, 7, 94448-94459. https://doi.org/10.1109/ ACCESS.2019.2928306

Zhu, Y., \& Gao, Z. (2014). Robust observer-based fault detection via evolutionary optimization with applications to wind turbine systems. In 9th IEEE Conference on Industrial Electronics and Applications (pp. 16271632). IEEE Publishing. https://doi.org/10.1109/ICIEA.2014.6931428 